\title{
O Uso da Distância de Hausdorff como Medida de Similaridade em Sistemas Automáticos de Atualização Cartográfica
}

\author{
The Use of Hausdorff Distance as Similarity Measure in Automatic Systems for \\ Cartographic Update
}

\section{Glauber Acunha Gonçalves ${ }^{1}$}

\section{Edson Aparecido Mitishita ${ }^{2}$}

1 Universidade Federal do Rio Grande - FURG - Centro de Ciências Computacionais

Av. Itália, km 8, s/n, Campus Carreiros, Rio Grande, RS, Brasil. Email: glaubergoncalves@furg.br.

2 Universidade Federal do Paraná - UFPR - Programa de Pós-Graduação em Ciências Geodésicas

Av Cel Francisco $\mathrm{H}$ dos Santos, s/n, CENTRO POLITÉCNICO, Bairro: Jardim das Américas, Curitiba, PR, Brasil. Email:mitishita@ufpr.br

\section{Resumo:}

A automação do processo de revisão e atualização da cartografia de áreas urbanas ainda constitui um desafio aos desenvolvedores de sistemas computacionais de alta produtividade. Uma das questões que permeia esse processo é a quantificação da similaridade entre objetos representados no espaço vetorial (bases cartográficas digitais) e segmentados no espaço matricial (imagens de alta resolução). A pesquisa desenvolvida no Laboratório de Fotogrametria do Departamento de Geomática da UFPR, ora apresentada, parte da premissa da existência de dados qualificados que permitam a segmentação precisa da imagem, a associação dos segmentos gerados às edificações erigidas sobre o terreno e a adequada georreferência dos dois conjuntos de informações. A partir desse resultado, o método proposto explora uma métrica topológica robusta, a Distância de Hausdorff, para determinação da condição de atualidade da representação cartográfica em função dos elementos presentes na imagem. Os testes de desempenho dessa métrica para uma área urbana com dados disponíveis demonstram uma alta taxa de concordância quando comparados os resultados com a fotointerpretação profissional e demonstrou-se que a medida é robusta à parcial obstrução dos objetos em comparação.

Palavras-chave: Distância de Hausdorff, Processamento de Imagens, Medida de Similaridade, Automação Cartográfica

\footnotetext{
Abstract:

The automatic process of reviewing and updating urban maps is still a challenge to developers of high productivity computer systems. One of the intrinsic issues of this process is to quantify the similarity between objects represented in the vector space (digital cartographic bases) and that
} 
segmented in the spatial matrix (high resolution images). The research developed in the Photogrammetry Laboratory of the Department of Geomatics (UFPR), presented here, assumes the existence of qualified aerial images, which provide the adequate segmentation and the association of these segments to the generated buildings, and the adequate georeference of both data sets. From this result, the proposed method exploits a robust topological metrics, the Hausdorff Distance, to determine the up to date condition of the cartographic representation of elements in the image. Performance tests of this metric shows a high rate of agreement when compared with professional photo interpretation, and has shown that the measure is robust to partial obstruction of the compared objects.

Keywords: Hausdorff Distance, Digital Imagem Processing, Similarity Measure, Automated Cartography

\section{Introdução}

Um sistema computacional, que objetive automatizar e acelerar o processo de atualização da cartografia de áreas urbanas, necessariamente será concebido para processar, com grande precisão, um determinado volume de informações gráficas em alta resolução. Parte-se do pressuposto de existir uma base cartográfica vetorial que represente as feições dos meios urbanos. E um conjunto de imagens fotogramétricas digitais, ou de sensores orbitais, e uma nuvem de pontos laser scanner, por exemplo, que permitam a segmentação precisa dos objetos edificados acima do nível do solo, como em Machado (2006).

Tal sistema, em algum ponto de seu conceito metodológico e, por consequência, de sua lógica de processamento, exigirá a utilização de uma métrica de similaridade, permitindo a tomada de decisão acerca da consistência dos registros vetoriais da base cartográfica, face a imagem em coerente posição geográfica.

Porém, o que é de simples decisão ao olhar humano, é complexo e sujeito a falhas pela análise computacional. Não se pode desconsiderar a capacidade de segmentar objetos da qual é dotado o sistema visual humano, com potencial de processar um conjunto complexo de parâmetros, como cor, textura e forma, vincular o resultado à semântica do espaço em análise onde os objetos se inserem, desprezando detalhes desnecessários, focando em bordas e maiores contrastes, resolvendo descontinuidades, associando o observado a formas pré-conhecidas, e tomando a decisão: o objeto da imagem é ou não aquele representado na base cartográfica. Para sistemas computacionais cada tarefa dessas é de complexa solução, vide McKeown Jr (1989) e Mayer (1999).

A Figura 1 é um exemplo desse processo de avaliação de consistência. No lote 'a' observa-se a conformidade do que se apresenta na base cartográfica pré-existente e os limites do que se observa como telhado na imagem. No lote 'b' percebe-se parcial consistência, visto que houve notado acréscimo à construção, pela diferença perceptível no telhado. Em 'c' onde não havia nada, agora aparece uma construção. O exemplo apresentado é produto da sobreposição dos vetores da base cartográfica sobre uma imagem, devidamente processada sob método fotogramétrico digital, ortorretificada e registrada sob mesmo referencial geodésico. Essa é a 
ação convencional e comumente executada para permitir a atualização das bases cartográficas cadastrais, ou seja, a fotointerpretação de imagens em ambientes de edição cartográfica digital.

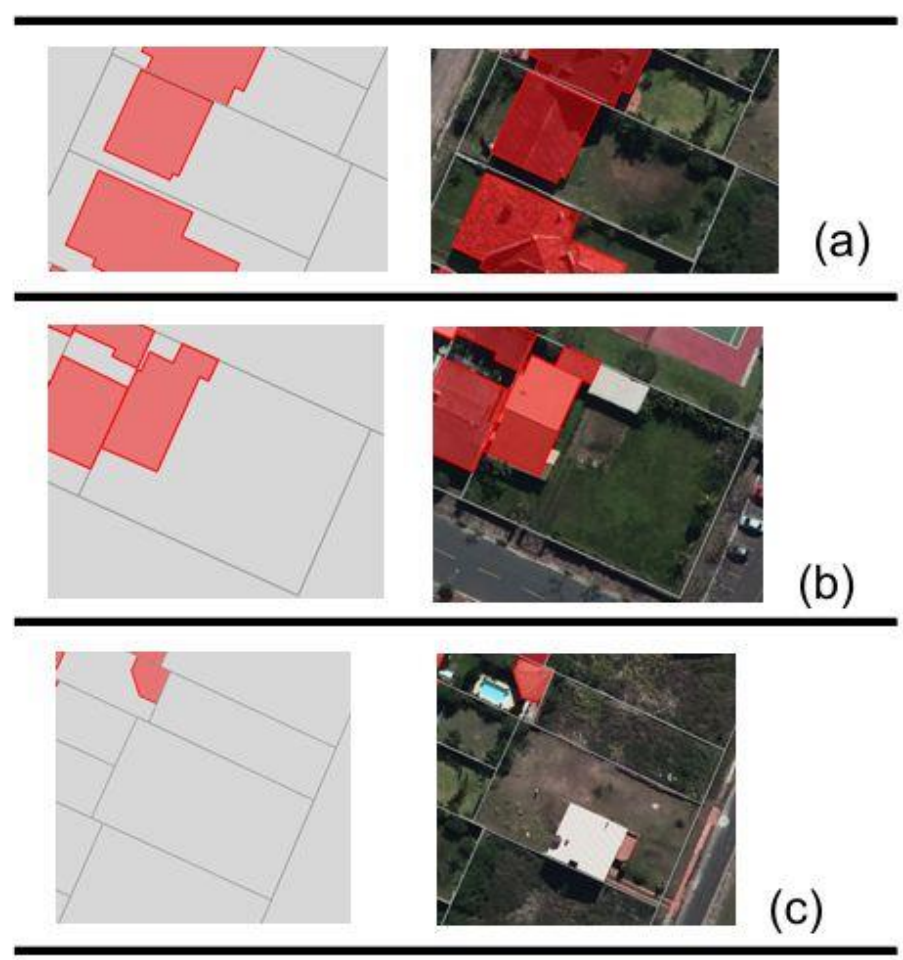

Figura 1: Três cenários na verificação de compatibilidade entre base cartográfica e imagens aéreas

Uma vez que a imagem esteja processada de forma a identificar objetos sobre ela, ou seja, uma vez que a imagem seja alvo de um conjunto de transformações que permite a conexão de seus pixels para definir regiões que podem ser associadas a objetos, resta rotular automaticamente se esses objetos segmentados, correspondem ou não, aos presentes na base vetorial. É nesse processo que se insere a metodologia aqui apresentada.

O objetivo da técnica é, pois, efetuar uma medida de similaridade entre geometrias, representadas em dois tipos de dados diversos, vetoriais e matriciais, com relativa robustez à pequenas variações de forma e, cujo resultado, possa quantificar o quanto tais geometrias representam ou não o mesmo objeto.

A distância de Hausdorff, definida para medir o quanto dois conjuntos de pontos dispersos em um espaço cartesiano são similares, constitui o fundamento desse método que será detalhado a seguir nos seguintes tópicos: a seção 2 apresenta um embasamento teórico dessa distância topológica e a aplicação da Distância de Hausdorff em um problema de medida de similaridade entre geometrias computacionais. A seção 3 caracteriza uma amostra para teste da métrica e o sistema desenvolvido para julgamento de seu desempenho em identificar objetos similares representados em espaços gráficos distintos. A seção 4 analisa os resultados obtidos pela aplicação da técnica ao conjunto de amostras. A seção 5 sumariza as conclusões desse estudo e apresenta futuros desenvolvimentos nessa linha de pesquisa. 


\section{Fundamentação e Aplicação da Métrica Topológica}

A medida de similaridade entre dois objetos no ambiente computacional é uma ação fundamental para desempenho de determinados sistemas automáticos. Seja na comparação de caracteres, expressões linguísticas, figuras e imagens, o problema de medir o quanto dois conjuntos binários são similares é um desafio constante aos desenvolvedores, considerando fatores como eficiência na medida e custo computacional.

Jain et al. (1995) inicia uma profícua discussão sobre as classes de medidas de similaridade, apontando para a importância da aplicação de lógica difusa, especialmente quando o objetivo é identificar uma imagem em um banco de imagens, dando origem a uma série de artigos sobre o problema. Na sequência, mais especificamente orientados para a questão do registro de imagens, algumas produções são apresentadas, como em Kalinic (2011) onde é analisado o desempenho de diversas distâncias nas medidas de similaridade entre amostras. E em Chinpanthana (2012) a medida de similaridade é parte de um processo maior que visa a interpretação semântica de imagens.

Definir o quando dois conjuntos são similares, em qualquer das situações citadas e na abordagem aqui presente, é um dos problemas centrais. As medidas de distâncias entre conjuntos podem ser abordadas de muitas formas, entre as mais convencionais, por exemplo, pela distância euclidiana entre as médias dos elementos dos conjuntos. Porém, em muitos casos, essas distâncias conferem uma noção de proximidade muito diversa daquela resultante da observação intuitiva humana e daquela que leva a interpretação adequada do processo em análise.

A evolução desse conceito leva a consideração de outros melhor elaborados, como a Distância de Hausdorff, que é apresentada como mais fidedigna na maioria dos casos e apresenta significativa concordância com diagnósticos visuais sobre tais problemas, visto o que foi demonstrado em Huttenlocher (1990).

A distância de Hausdorff (HD), como apresentada por Gregoire et al. (1998), deve sua definição aos exercícios desenvolvidos por Felix Hausdorff, e têm sido utilizada por grupos de visão computacional, como em Hunttenlocher e Kedem (1990) e Hunttenlocher et al. (1992, 1993).

Originalmente HD é definida como:

$$
\begin{aligned}
& H(A, B)=\max \{h(A, B), h(B, A)\} \\
& h(A, B)=\max _{a \in A}\left\{\min _{b \in B}\{d(a, b)\}\right\} \quad h(B, A)=\max _{b \in B}\left\{\min _{a \in A}\{d(b, a)\}\right\}
\end{aligned}
$$

$\mathrm{A}=\{a 1, a 2, \ldots a m\}$ e $\mathrm{B}=\{b 1, b 2, \ldots b n\}$ são dois conjuntos com número finito de pontos e $\mathrm{d}$ é uma distância entre dois pontos, por exemplo, a distância Euclidiana ou a distância Manhatan, no caso de espaços matriciais. O significado dessas distâncias é simples. O algoritmo para determinação de HD também é de simples compreensão:

- Toma-se um elemento de A e calculam-se as distâncias a todos os elementos de B. Registra-se o menor valor dentre esses calculados;

- Repete-se o passo anterior para todos os demais elementos de A;

- Determina-se o maior valor registrado.

Ou seja, $\mathrm{h}(\mathrm{A}, \mathrm{B})$ é a maior das menores distâncias que separam os pontos de $\mathrm{A}$ de qualquer ponto de $\mathrm{B} ; \mathrm{h}(\mathrm{B}, \mathrm{A})$ será determinada por processo idêntico, somente considerando sentido inverso para o computo das distâncias. É importante relembrar que as operações de máximo e mínimo não são 
simétricas, portanto devem ser orientadas, donde $\mathrm{h}(\mathrm{A}, \mathrm{B})$ pode, e normalmente, não será igual a $\mathrm{h}(\mathrm{B}, \mathrm{A})$.

A HD será a maior entre as duas $\mathrm{h}(\mathrm{A}, \mathrm{B})$ e h(B,A). A Figura 2 apresenta graficamente o conceito. Evidentemente, para qualquer ponto tomado em um dos conjuntos, haverá um ponto do outro conjunto afastado no máximo H(A,B) e esse é o significado topológico da HD.

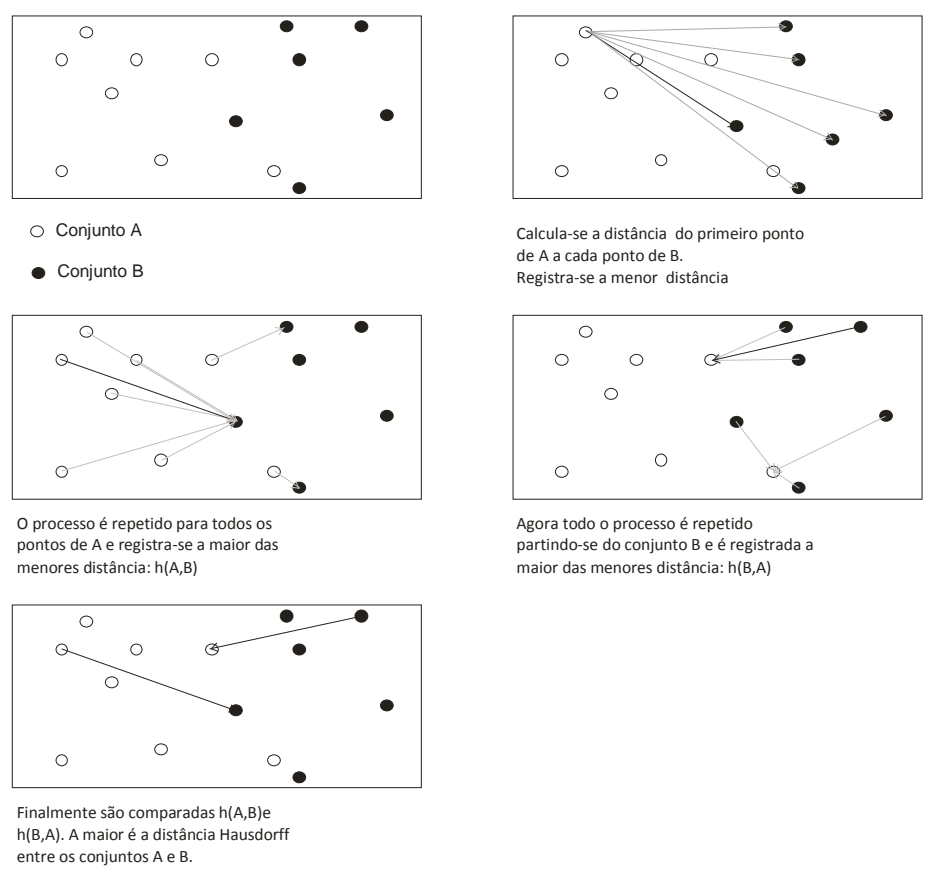

Figura 2: A definição da Distância Hausdorff

Quando relacionada a objetos, descrito por seus vértices, a aplicação dessa técnica exige alguma generalização, análise matemática e o uso de artifícios computacionais para permitir viabilidade de bons resultados em tempo de processamento compatível. Por exemplo, a distância entre dois polígonos simples e convexos, que não se interceptam, e onde um não contém nem está contido no outro, é resolvida pelo axioma de Grégoire (1998): a HD entre os polígonos sempre será a HD entre o conjunto de vértices que formam o polígono.

Porém, quando o interesse é utilizar a métrica para comparar objetos complexos, com posições e interferências espaciais, a abordagem exige maior rigor e essa condição simplificada não será verificada. Nesse caso a HD deve ser determinada considerando a distância entre as linhas que delimitam o polígono.

A Figura 3 ilustra a aplicação do princípio ao caso de uma forma base que é comparada com duas outras concorrentes. A Figura $3 a$ é a forma base, que será comparada com $3 b$ e $3 c$ a fim de determinar qual é mais similar. Em 3d e 3e mostra-se a superposição das formas, indicando a Distância de Hausdorff entre elas. Um algoritmo de convergência baseado na HD, responde a questão. A forma cuja HD é menor será escolhida como mais similar à forma procurada.

A utilização de métricas como a área, perímetro, fator de forma, compacidade, posição do centróide, entre outras, não atuaria de forma mais qualificada e, provavelmente, não conduziria a um julgamento tão confiável, além do que a composição de múltiplos indicadores exige um fator de decisão mais complexo. Pesa contra o uso da HD, no entanto, o alto consumo de tempo de processamento, isso em função de geometrias descritas por muitos vértices.

A forma geométrica pode estar também representada num espaço matricial, como pixels numa imagem binária, conforme já foi escrito. Nesse caso o computo de HD volta a envolver somente 
a distância entre pontos, porém, contidos numa grade regular, registrada segundo referencial comum, permitindo a otimização o tempo de processamento.

A Figura 4 ilustra o problema e a análise a ser produzida para sua solução.

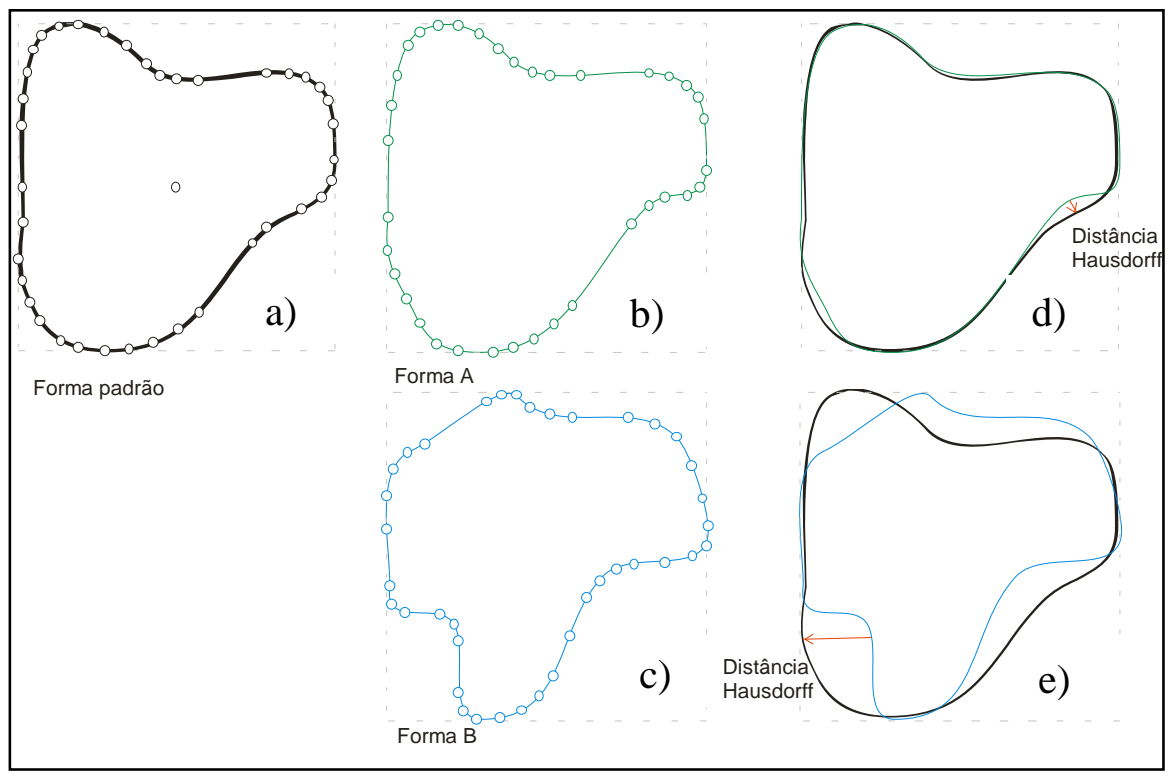

Figura 3: A Distância Hausdorff entre dois polígonos

a)

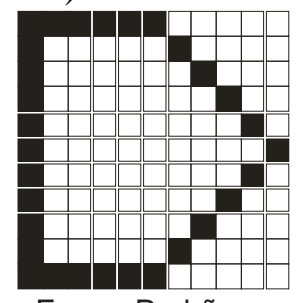

Forma Padrão b)

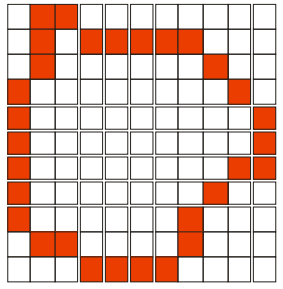

Forma A

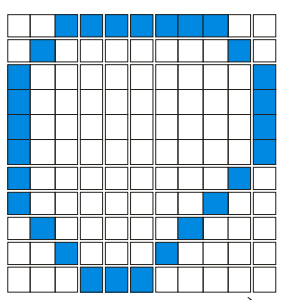

Forma B
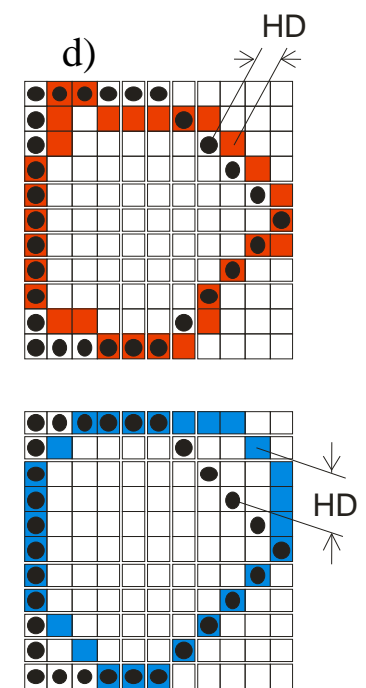

e)

Figura 4: A Distância Hausdorff entre dois polígonos em suas representações matriciais

$\mathrm{Na}$ Figura 4, em 4a, está a forma padrão e em 4b e 4c as formas concorrentes. Em 4d e 4e a superposição das Figuras anteriores no mesmo espaço amostral. Observe-se que a forma A não possui nenhum de seus pixels afastados mais do que um pixel da forma padrão, enquanto a forma B possui um pixel cuja mínima distância é de 3 pixels aos da forma padrão, no caso considerando a denominada distância city-block, conforme Gonzales e Woods (2007) (também conhecida como Manhattan ou taxi-cab, determinada pelo afastamento entre dois pixels sem permitir deslocamentos diagonais). Ou seja, a Distância Hausdorff entre a forma padrão e a forma A vale um pixel e entre a forma padrão e a forma B três pixels. Assim, a forma A é eleita 
como a melhor representação do padrão, o que concorda com a observação visual das geometrias.

Uma questão importante na aplicação dessa métrica é o custo computacional. Na sua forma elementar, o cálculo de HD exigiria $\left(\mathrm{Np}^{*} \mathrm{Nq}\right)$ operações de determinação de distância entre dois pixels, sendo $\mathrm{Np}$ o número de pontos da forma padrão e $\mathrm{Nq}$ o número de pontos da outra forma em teste. Isso representa um alto custo computacional. Mas pode-se otimizar esse procedimento com o uso do algoritmo da superfície de Voronoi, segundo Paglieroni (1992).

Considere-se a função $d(x)$ como segue:

$$
d(x)=\min _{p=P}\|x-p\|
$$

onde $\mathrm{P}$ é um conjunto finito de pontos em $\mathrm{R}^{2}$ e o domínio de $\mathrm{X}$ é $\mathrm{R}^{2}$. $\mathrm{O}$ gráfico da função d(x) é uma superfície que tem sido chamada superfície de Voronoi e representa a distância ao ponto $\mathrm{p}$ mais próximo contada desde cada ponto $x$. Essa superfície possui a forma de vários cones justapostos (similares a uma embalagem para armazenar ovos) onde os mínimos valem zero e estão exatamente sobre cada p pertencente a P. Seus máximos estão na interceptação dos cones e sempre equiidistantes de dois ou mais pontos $\mathrm{p}$. A projeção desses máximos sobre o plano $\mathrm{d}=0$ é conhecida como diagrama de Voronoi e correspondem às posições eqüidistantes de dois ou mais pontos de um conjunto.

A vantagem da abordagem baseada na construção da superfície de Voronoi é que para uma grade equiidistante, como numa imagem digital, Borgefors (1986) apresenta um algoritmo bastante simples para sua solução, numa operação denominada transformada de distância:

i) Dado uma imagem binária (img) onde os pixels objeto valem um e o fundo zero;

ii) Criar uma imagem temporária (img_temp) com todos os pixels iguais a infinito positivo, exceto nas posições onde img vale um, para as quais img_temp será feito zero. Infinito positivo pode ser obtido pela classe Inf definida pelo IEEE e disponível na maioria das linguagens computacionais de alto nível ou por um valor suficientemente alto, maior que o número de pixels da imagem, por exemplo.

iii) Para cada pixel de img_temp, desde o extremo superior esquerdo até o inferior direito testar:

- se o valor img_temp é diferente de Inf, e se for:

- se o valor do pixel vizinho a direita é maior do que (img_temp + 1) e se for fazêlo igual a (img_temp +1$)$; lo igual a (img_temp + 1);

- se o valor do pixel vizinho abaixo é maior do que (img_temp + 1) e se for fazê-

iv) Para cada pixel de img_temp, desde o extremo inferior direito até o superior esquerdo testar:

- se o valor img_temp é diferente de Inf, e se for:

- se o valor do pixel vizinho à esquerda é maior do que (img_temp + 1) e se for fazê-lo igual a (img_temp + 1);

- se o valor do pixel vizinho acima é maior do que $\left(i m g \_t e m p+1\right)$ e se for fazê-lo igual a (img_temp + 1); 
Na Figura 5, primeiramente, em 5a uma configuração de pixels-objeto é apresentada. Segue em $5 \mathrm{~b}$ a aplicação do algoritmo acima (a transformada de distância) e por fim, em 5c, o diagrama de Voronoi para o mesmo conjunto.

(a)

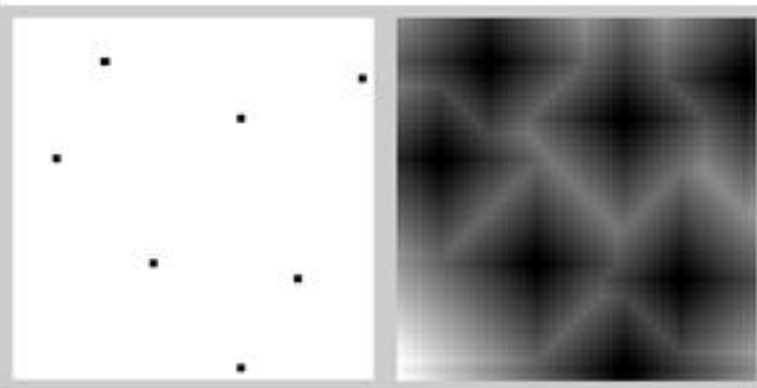

(c)

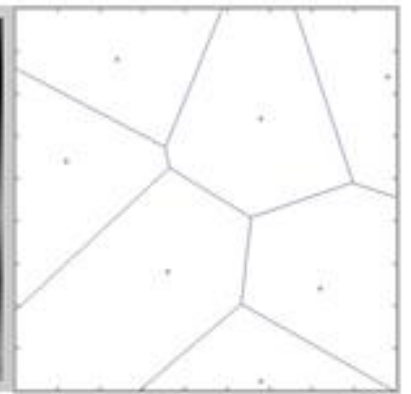

Figura 5: A definição gráfica da Transformada de Distância e do Diagrama de Voronoi (em $a$, um conjunto discreto de pontos, em $b$ a transformada de distância onde os tons mais claros corresponde a valores mais altos e, em $c$, o diagrama de voronoi com a projeção no plano dos máximos da $b$ )

Uma vez aplicada à transformação de distância em todo o domínio da imagem, ou das imagens no caso da comparação de dois objetos, a HD torna-se uma operação de ordenamento de distâncias. Se A e B são as imagens binárias a serem comparadas e d(A) e d(B) as respectivas transformadas de distância, então a determinação de HD faz-se tomando o valor d(A) nas posições correspondentes ao objeto de $\mathrm{B}$ e determinando-se o maior valor. Repete-se a operação considerando os valores $\mathrm{d}(\mathrm{B})$ nas posições que definem $\mathrm{A}$. O maior dentre os dois máximos encontrados será HD, como representado na Figura 6.

a)

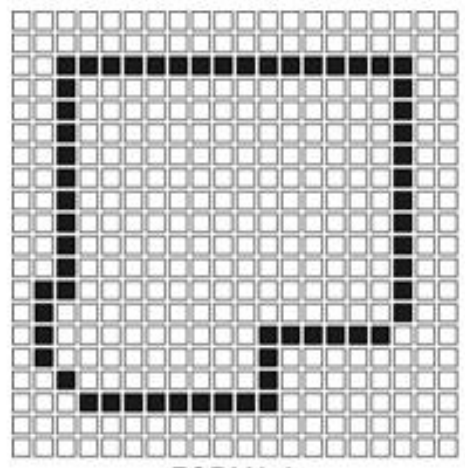

FORMA A

c)

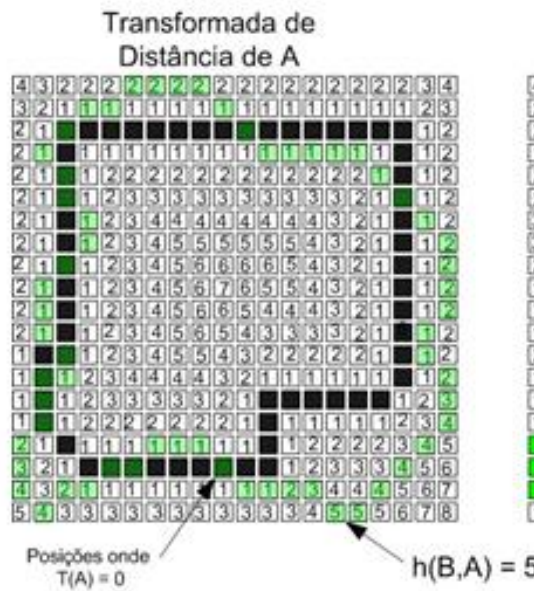

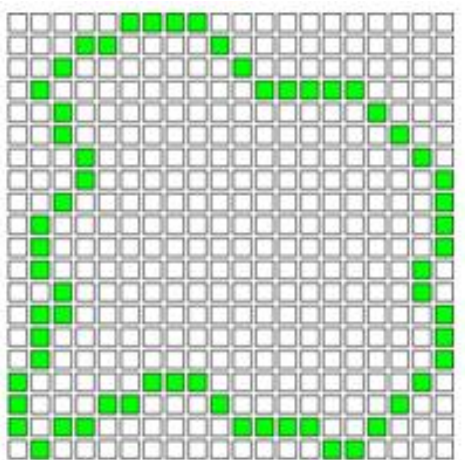

FORMA B

Transformada de

Distância de $\mathrm{B}$

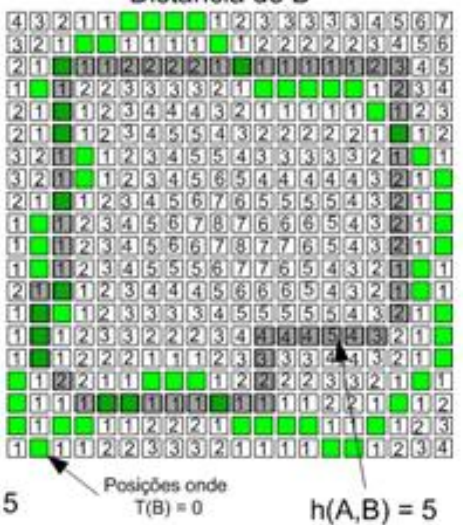

b)

d)

Figura 6: Cálculo da Distância de Hausdorff pela Transformada de Distância 
Considere uma imagem binária, como as mostradas em $6 \mathrm{a}$ e $6 \mathrm{~b}$ na Figura acima, de 20 por 20 pixels, contendo alguns pixels objeto (em preto e verde, respectivamente) sobre um background padrão (em branco). A imagem $6 \mathrm{c}$ é a transformada de distância de $6 \mathrm{a}$, ou seja, os valores representam a distância mínima de cada um dos pixels à forma A. Evidentemente, os pontos marcados em preto em 6c correspondem a valores nulos, uma vez que se está sobre a própria forma A. Equivalente operação é mostrada em $6 \mathrm{~d}$ para a forma B.

Então, projetando a forma A sobre 6d e a forma B sobre 6a e aparecem os pontos em destaque, cujos valores serão utilizados para o cálculo de HD. Resta determinar o maior dos valores em 6c e $6 \mathrm{~d}$ e escolher o maior dentre eles: $\operatorname{assim}, \mathrm{h}(\mathrm{A}, \mathrm{B})=5 \mathrm{e} \mathrm{h}(\mathrm{B}, \mathrm{A})=5$, então, $\mathrm{HD}=5$.

Uma alternativa mais robusta para comparar a forma de objetos em imagens complexas, como no caso de aerofotos de áreas urbanas, onde interferências e obstruções podem estar presentes, é utilizar um índice baseado no número de pontos dos conjuntos que obedecem a uma condição de vizinhança, conceitualmente similar ao princípio de HD.

Essa é a proposta de Orrite (2004), aqui descrita como VHD, numa alusão à uma faixa de vizinhança determinada pela distância de Hausdorff.

Seja hA o conjunto de pontos onde é válida a condição $\{\min \|b-a\|<P\}$ e hB o correspondente conjunto onde $\{\min \|a-b\|<P\}$. O valor $\mathrm{P}$ é a máxima distância para que um ponto seja considerado suficientemente próximo ao outro a fim de computar VHD. Sendo KA o número de pontos que satisfazem à primeira condição, KB o correspondente para a segunda condição, e dados p e q como os respectivos tamanhos dos conjuntos A e B, então:

$$
h_{v}(A, B)=\frac{K_{A}}{p} \quad \mathrm{e}^{h_{v}(B, A)=\frac{K_{B}}{q}}
$$

E, por conseguinte:

$$
\operatorname{VHD}(A, B)=\min \left(h_{v}(A, B), h_{v}(B, A)\right)
$$

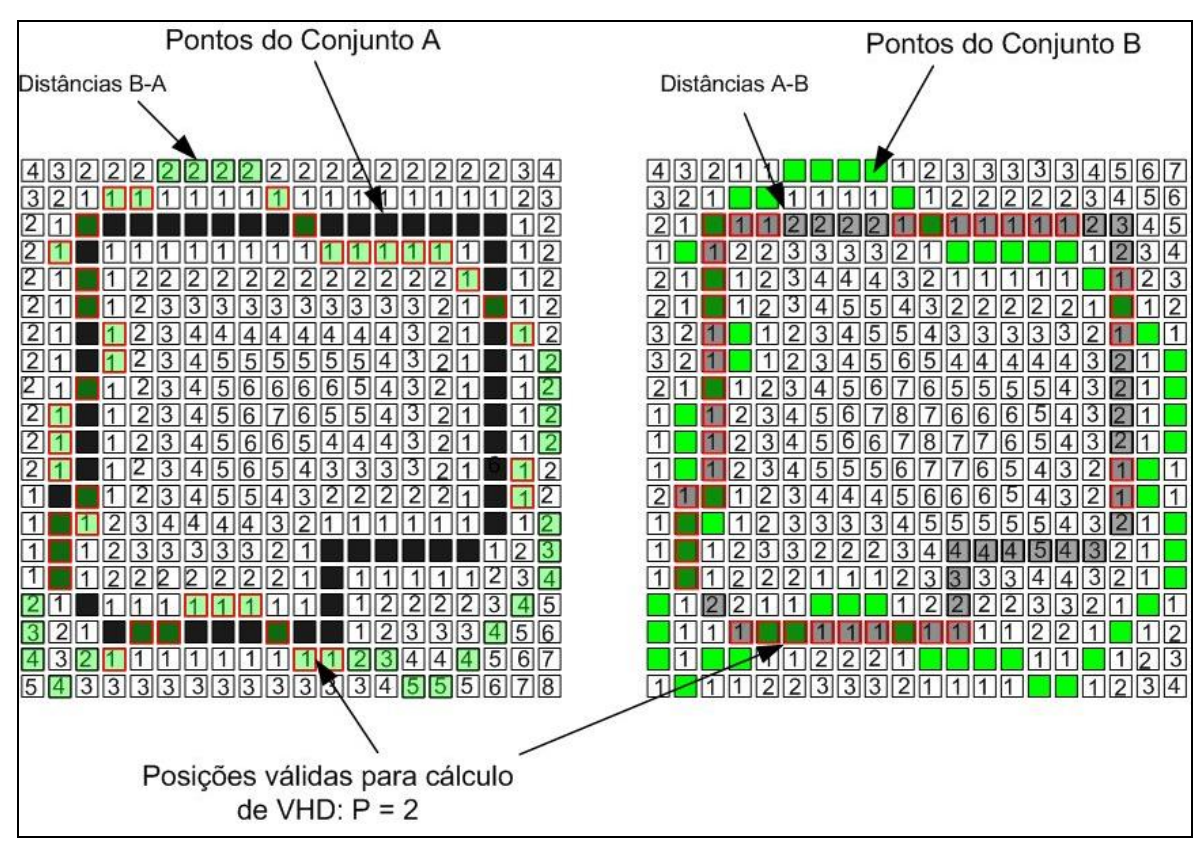

Figura 7: Cálculo da Distância de Hausdorff pela Transformada de Distância 
A Figura 7 ilustra esse procedimento, tomando a mesma arte das Figuras $6 \mathrm{c}$ e $6 \mathrm{~d}$. A Figura A possui 59 pontos ativos (aqueles com valores destacados em sombreamento verde e cinza, respectivamente) e a Figura B 61 pontos, donde, $p=59$ e q=61. Arbitre-se P igual a 2 pixels, ou seja, a distância máxima aceitável entre os dois conjuntos de pontos para computo de VHD será 1 pixel. Então, contando o número de pontos cuja distância A-B atende a condição estabelecida (marcados com contorno em vermelho) chega-se a 38 e o número de pontos cuja distância B-A, sob mesma restrição, e encontra-se, por coincidência do exemplo, também 38. Assim sendo, $\mathrm{hv}(\mathrm{A}, \mathrm{B})=(38 / 59)=0,644 \mathrm{e} \mathrm{hv}(\mathrm{B}, \mathrm{A})=(38 / 61)=0,623$. VHD, portanto, assumirá o menor desses valores, ou seja, $\operatorname{VHD}(\mathrm{A}, \mathrm{B})=0,623$.

Será esse o procedimento utilizado para determinação da condição de similaridade do experimento apresentado na próxima seção, onde os objetos alvo são aqueles pré-existentes na base cartográfica e os objetos para comparação são aqueles resultantes da segmentação da imagem fotogramétrica adquirida para atualização dessa base.

\section{Material e Métodos}

Para teste da técnica foram selecionados um conjunto de dados de 187 lotes, presentes em 12 quadras da área urbana de uma cidade do litoral do estado do Paraná. Ou seja, a expressão matricial da verdade de campo no presente, a imagem fotogramétrica, e a expressão vetorial do que foi aquele local em data anterior, a base cartográfica. $O$ teste de similaridade pode assim determinar a condição de obsolescência da base cartográfica em relação as edificações ali existentes, diante das imagens aerofotogramétricas adquiridas.

A Figura 8 ilustra a técnica utilizada na extração das amostras para o teste de similaridade. As coordenadas dos vértices dos lotes e das construções (8b) são extraídas da base cartográfica (8a). Os limites dos lotes, em coordenadas geodésicas, são então utilizados para recorte da área do mesmo sobre a imagem aerofotogramétrica ortorretificada e devidamente georreferenciada (8c). A imagem resultante ( $8 \mathrm{~d})$ segue processamento clássico: é segmentada por suas cores pelo algoritmo Mean Shift, conforme Gu, I.Y.H.; Gui, V. (2001) e Comaniciu e Meer (2002) (8e); e classificados os seus segmentos como pertencente a classe telhados ou não (8f), conforme descrito por Gonzalez (2007), por Jensen (1996) e por Somboonkaew et al. (1999). Para identificação dos segmentos que são coberturas de edificações, amostras de elevações obtidas sobre nuvem de pontos obtidas por scanner laser são fundamentais.

Assim dispõem-se de duas imagens cujas coordenadas são relativas ao mesmo sistema de referência e amostradas segundo a mesma resolução: uma resultante da aeroimagem classificada, aqui denominada I1, e outra, sintetizada a partir dos dados da geometria presente na base cartográfica, I2. A Figura 9 mostra essas duas imagens. Uma descrição mais detalhada desse processo pode ser vista em Gonçalves (2007).

Segue, pois, a aplicação da transformada de distância sobre I1 e I2, resultando Dist(I1) e Dist(I2). Sobre Dist(I1) seleciona-se todos os pontos nas posições onde está a geometria I2 resultando $\mathrm{V}(1,2)$ e sobre Dist(I2) os pontos onde está a geometria de I1, donde teremos $\mathrm{V}(2,1)$. $\mathrm{V}(1,2)$ e $\mathrm{V}(2,1)$, como dito na seção anterior, são dois vetores com as máximas das mínimas distâncias que separam as duas geometrias, medidas pixel a pixel, no espaço discreto onde estão definidas. 


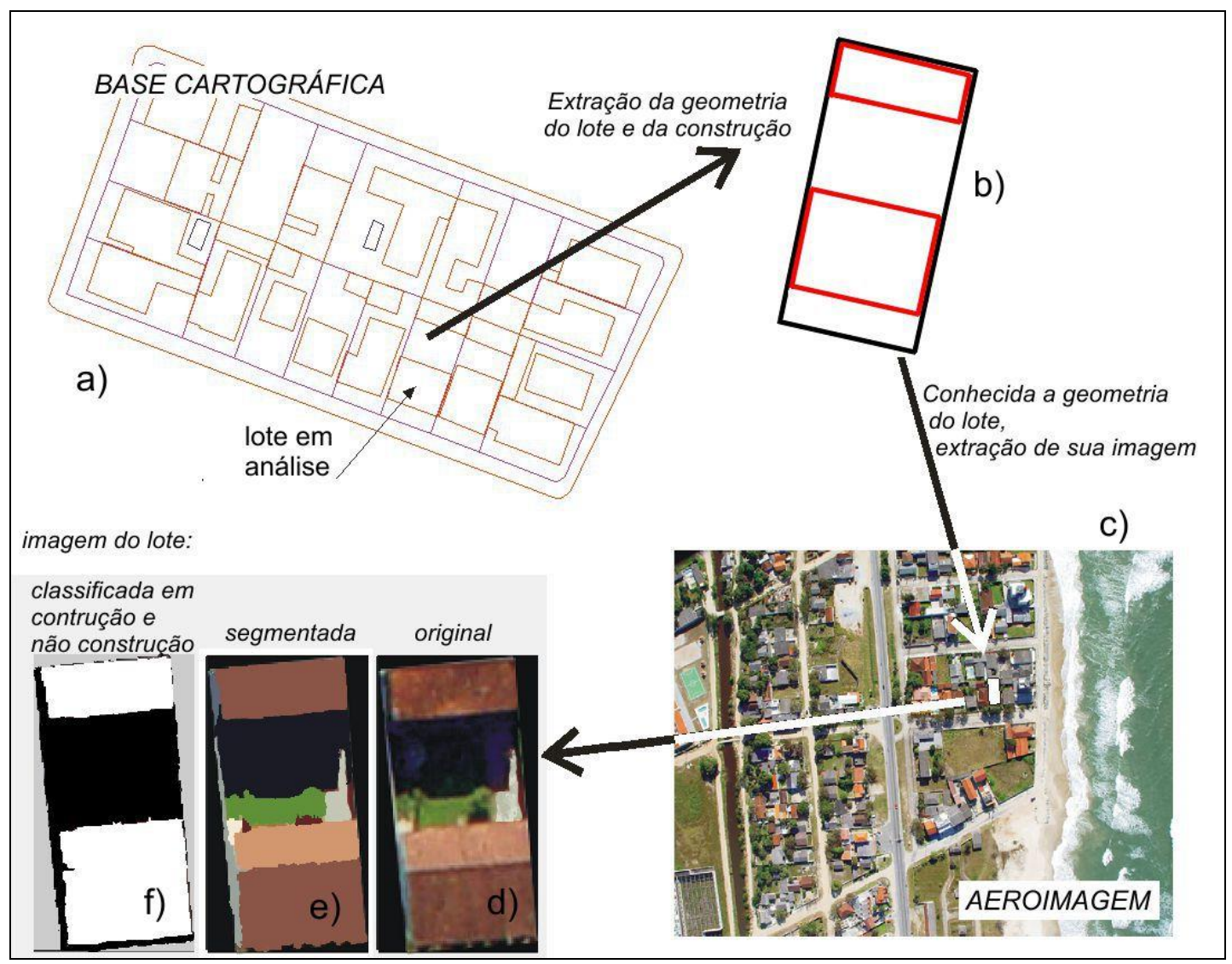

Figura 8: A técnica de preparação das amostras para teste da métrica de similaridade

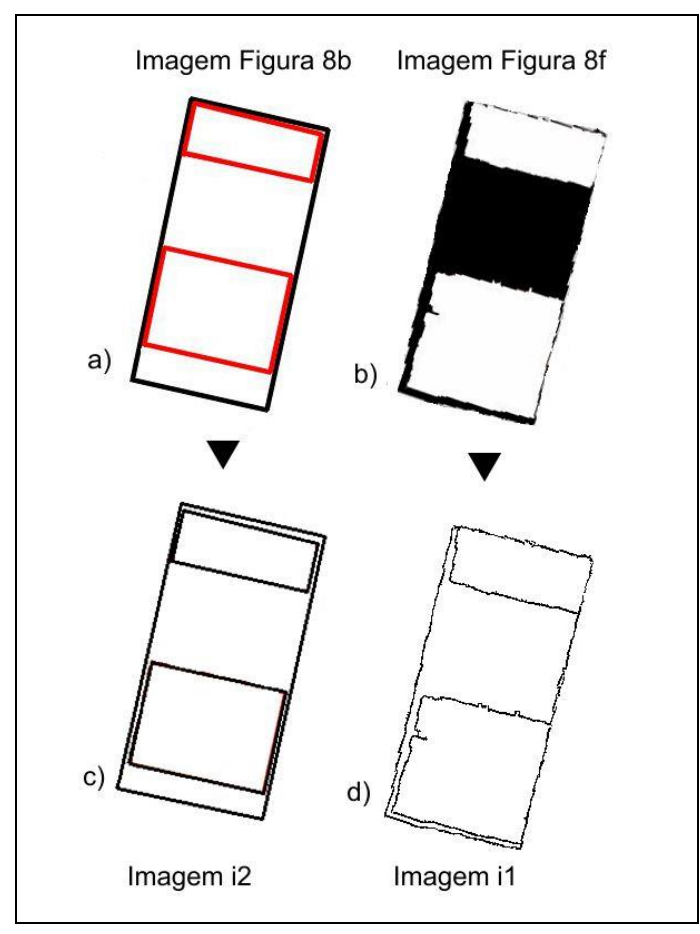

Figura 9: As imagens I1 e I2, resultantes do processamento da imagem aerofotogramétrica e da base cartográfica, respectivamente, e prontas para aplicação da métrica de similaridade 
Resta ordenar os vetores, determinar quantos dos valores em $\mathrm{V}(1,2)$ e $\mathrm{V}(2,1)$ estão abaixo de uma tolerância, a priori especificada, e calcular hv(1,2) e hv(2,1). O menor desses valores será a medida de similaridade entre os dois objetos. No experimento descrito na próxima seção esse limite foi arbitrado igual a 3 pixels, o que em unidades do espaço objeto significam $37,5 \mathrm{~cm}$ (taxa amostral da imagem em unidades de terreno $=0,125 \mathrm{~m}$ ). Esse valor guarda relação com o que se avalia significativo nos cenários de cadastros urbanos com fins tributários, por exemplo.

A definição da condição de atualidade ou obsolescência da informação contida no lote será proporcional ao valor dessa métrica. Valores próximos a unidade indicam alta compatibilidade entre as geometrias dentro da tolerância de 3 pixels parametrizada a priori. Valores muito baixos, próximos zero, são indicadores de que os pontos que definem os objetos em comparação estão separados por distâncias maiores do que a tolerância e, portanto, são diferentes.

Num sistema de decisão operacional, essa métrica pode ser configurada a fim de apresentar robustez a obstrução parcial dos objetos. Ou seja, para avaliar a similaridade a partir de uma maior parcela de pontos não obstruídos de certo objeto, ao mesmo tempo desprezando parcela de pontos onde ocorra um obstrução. Obviamente, um critério subjetivo acerca da significância dessa diferença estará sendo utilizado.

Por exemplo, no processo final de classificação do objeto como estando ou não compatível com a cartografia apresentada, limites para VHD podem ser estabelecido. Se, por suposição, tomar-se um valor mínimo de VHD > 0,80, ficará arbitrado que mesmo se $20 \%$ dos pontos estiverem fora da tolerância, ainda assim o objeto será dado como compatível. Ou ainda, 20\% da borda de um telhado pode estar obstruída pela copa de uma árvore, e ainda assim, se o restante estiver dentro da tolerância em pixels, o objeto será dado como similar.

Num sistema produtivo essa variável poderá ser controlada dinamicamente, assumindo valores mais restritivos, próximos a unidade, em áreas onde a probabilidade de obstruções for menor, como aquelas regiões das cidades onde é rara a presença de arborização. E valores de tolerância menores, como $75 \%$, onde sabe-se a priori da maior probabilidade de obstrução parcial dos telhados.

Evidentemente, uma avaliação sobre o que é efeito de obstrução e o que é realmente alteração da edificação deve ser sobreposta a essa medida.

\section{Resultados e Discussão}

Para teste da hipótese de consistência no uso da Distância de Hausdorff na medida de similaridade entre as feições presentes em uma base cartográfica em grande escala e àquelas passíveis de identificação por segmentação de imagens aéreas de alta resolução, foram selecionados alguns cenários específicos. São lotes de uma zona urbana no litoral do estado do Paraná, mais especificamente na cidade de Matinhos. Submetidos à rotina de processamento detalhada na seção anterior, conforme mostra a Figura 10, apresentam resultados como os indicados na tabela 01 .

A Figura 10a é o registro presente na base cartográfica. Por esse registro, cujos vértices do terreno podem ser facilmente extraídos, é orientado o recorte da imagem ortorretificadas (10b). Segue o processamento da imagem pela sua segmentação (d) e classificação entre as classes edificação e não edificação (e). Um simples detector dos limites entre as classes leva ao contorno dos objetos identificados na imagem (f), que materializa a imagem I1 descrita na metodologia. Ainda, do registro cartográfico (10a) sintetiza-se a imagem I2 (g). Na Figura apresenta-se a 
transformada de distância para I1 e sobre ela os pontos para cálculo de $\mathrm{V}(1,2)$, pela simples sobreposição de I2 (10h).

A segmentação das imagens foi executada através do uso do código disponibilizado por Comaniciu e Meer (1997), cuja função processa a imagem colorida em três bandas, convertida para o espaço de cores Lab e com parâmetro de segmentação "q" (quantization), recomendado pelos autores para imagens de alta resolução. Os resultado foram de qualidade suficiente para que, uma vez agregadas as amostras de altitudes provenientes de um vôo laser scanner, definissem os contornos das edificações, passíveis de serem utilizados na comparação com os dados da base cartográfica.

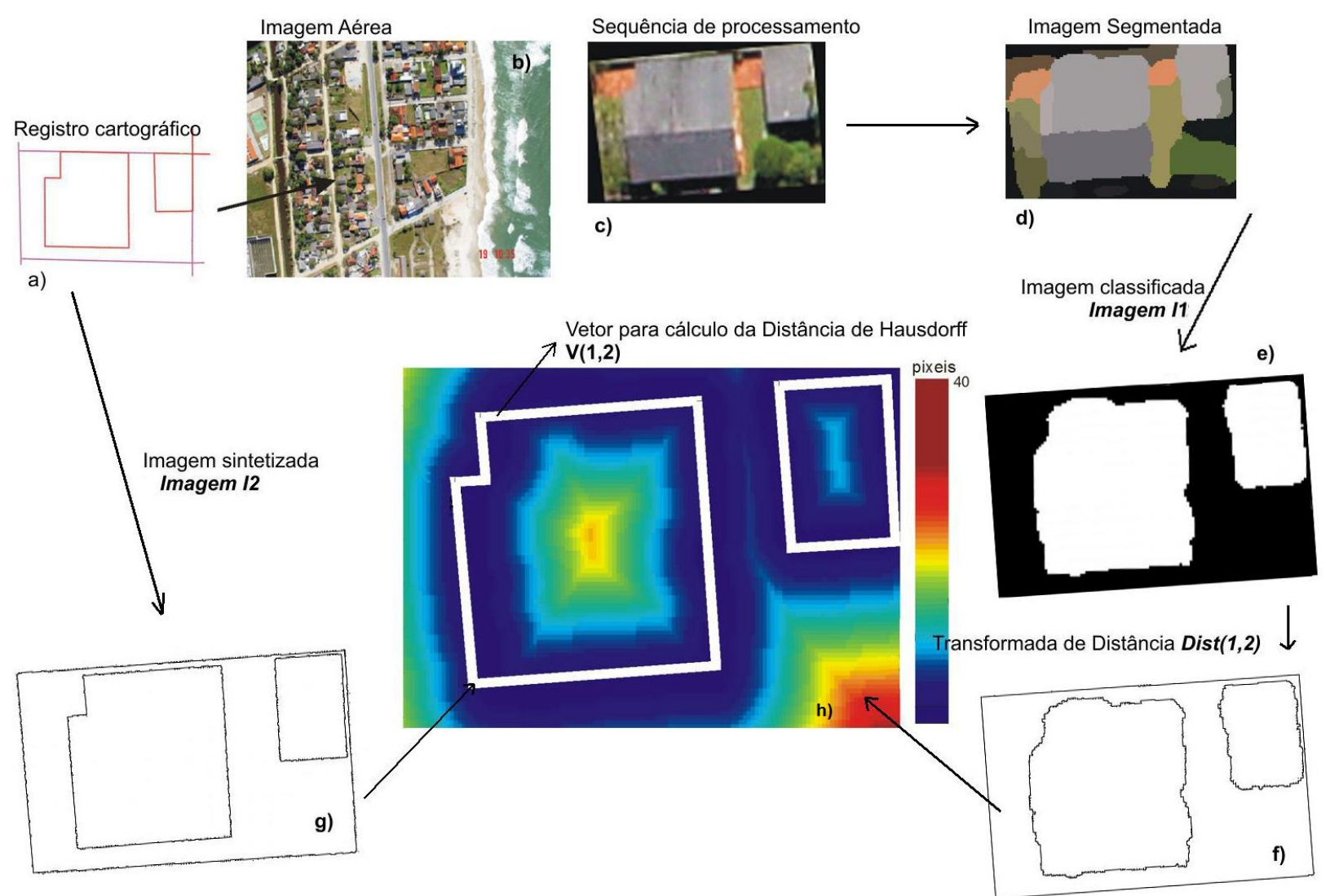

Figura 10: Aplicação da métrica de similaridade pela Distâncias de Hausdorff

Para teste da metodologia, e somente para esse fim, foram arbitrados os valores 3(três) pixeis, para a máxima distância utilizada no cálculo de VHD, e 0,8 (80\%), para limite de aceitabilidade da condição de coerência entre os cenários cartográfico e aerofotogramétrico. Num caso prático, um operador do sistema deve estar apto a selecionar tais parâmetros e, dessa forma, tornar o identificador de alterações mais, ou menos, tolerante às diferenças observadas.

Os três primeiros exemplos da tabela 01 são casos onde os valores de VHD são maiores do que 0,8 e, conforme se observa, correspondem a situações onde a análise visual indica lotes com construções igualmente presentes na base cartográfica e na imagem. Os exemplos 04 e 05 são casos onde VHD assume valores menores do que 0,5 , indicando situações onde apenas parte da construção originalmente registrada na base cartográfica está presente na imagem. A fotointerpretação da imagem recortada efetivamente indica que há construções que se alteraram no período em análise, ainda mais, em ambos os casos houve acréscimo nas áreas construídas, adjuntas às existentes.

Quando aplicada a um conjunto de 187 lotes, existentes em 12 quarteirões, conforme mostra a Figura 11, 95 resultaram em medidas de similaridade que indicam compatibilidade entre os 
registros cartográficos existentes. A interpretação visual de cada lote resulta na indicação de que o sistema acertou 93 dos diagnósticos de compatibilidade. Outros 92 resultaram em medidas de VHD menor do que 0,8 , o que levaria a interpretação de alterações significativas nas edificações sobre tais lotes. No entanto, apenas 42 lotes efetivamente haviam se alterado, ou seja, ocorreram 50 falsos positivos para alterações efetivas, casos relacionados a obstruções parciais do objeto por vegetação, obstrução por prédios vizinhos ou imperfeições na segmentação/classificação dos objetos associados aos telhados.

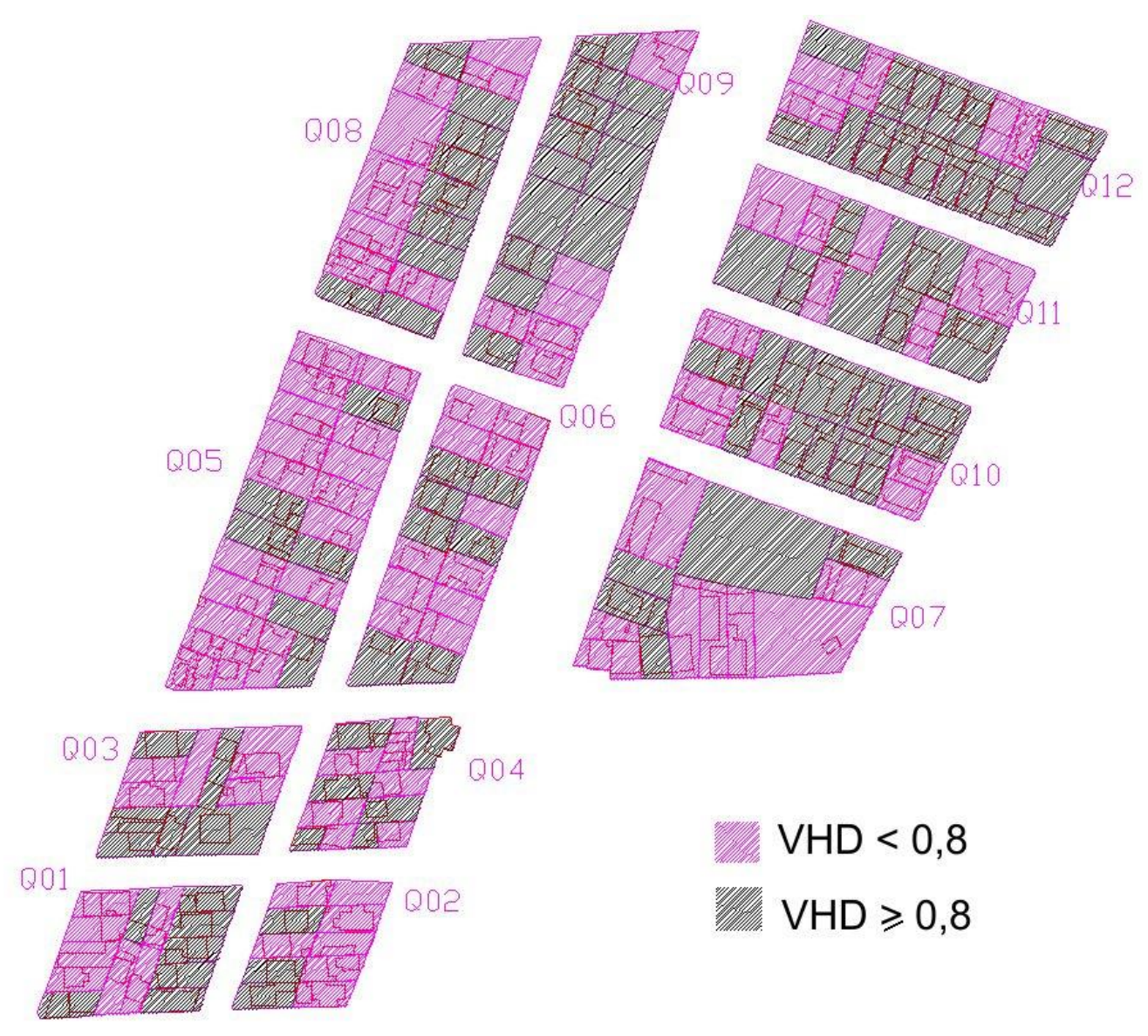

Figura 11: Área submetida à métrica de similaridade. No estudo de caso, adotado o limite arbitrado de $\mathrm{VHD}=0.8$, os lotes em preto apontam diferenças significativas e em magenta diferenças não significativas, ao nível de tolerâncias adotado 
Tabela 1: Algumas amostras da aplicação da métrica de similaridade baseada na Distância de Hausdorff

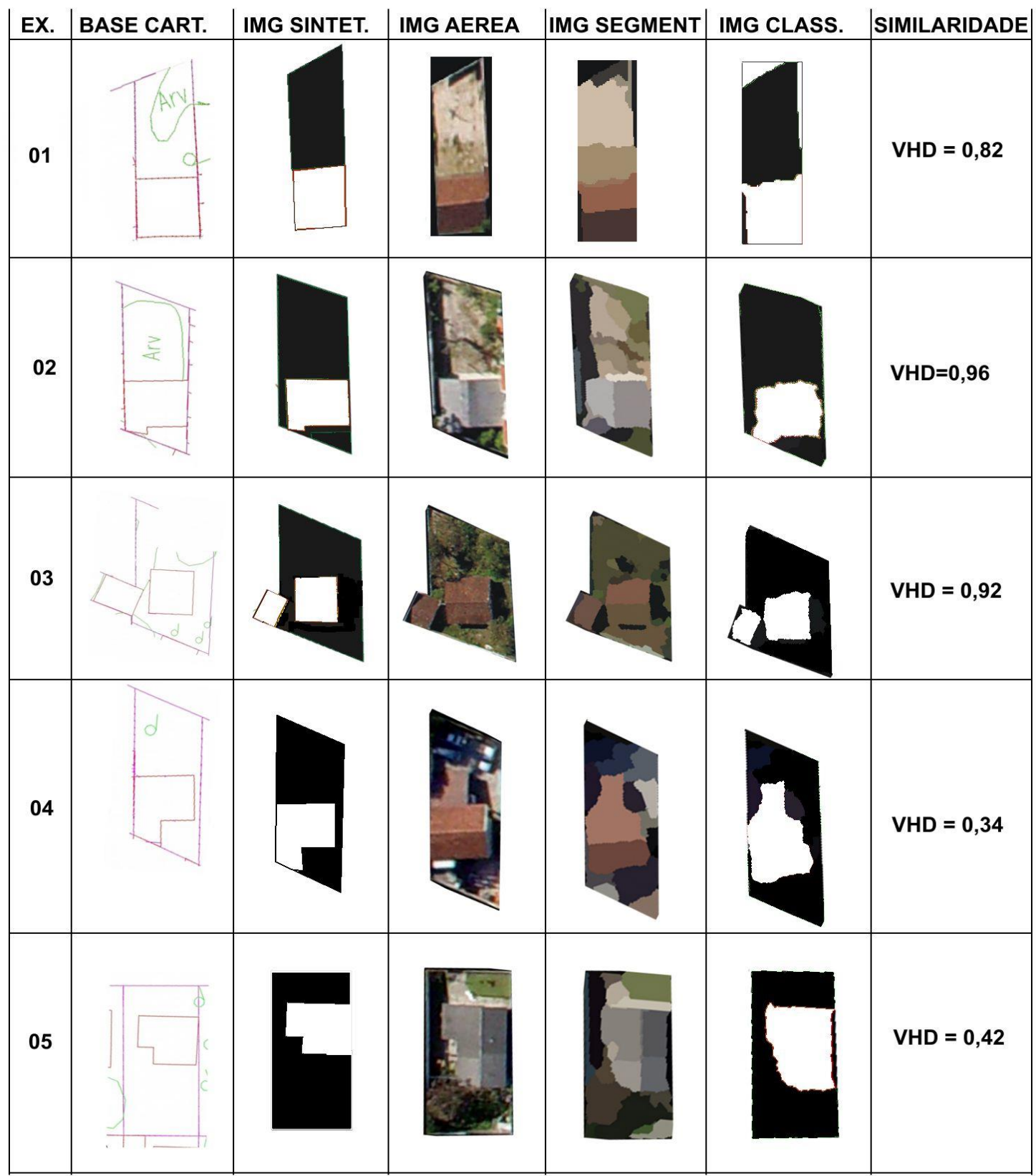

\section{Conclusões}

As métricas de similaridade exercem função de grande importância na interpretação de imagens, especialmente em casos de cenas com alta complexidade, onde os objetos provavelmente presentes apresentam significativa variância de suas propriedades visuais (cor, textura e forma).

A Distância de Hausdorff foi inicialmente proposta para analisar conjuntos de pontos isolados. Posteriormente mostrou-se adequada para estabelecer similaridade entre geometrias vetorialmente descritas. Por extensão, também se articula para resolver a similaridade entre feições descritas matricialmente em sistemas computacionais. É uma alternativa importante para 
o processamento de casos em que bases cartográficas cadastrais urbanas são colocadas em diagnóstico de obsolescência por imagens aerofotogramétricas.

No estudo de caso apresentado nesse artigo uma metodologia é apresentada e implantada em ambiente computacional interativo. De uma amostra de 187 lotes o sistema identificou 95 em condições de similaridade, $98 \%$ de forma correta. O sistema, por consequência, identificou 92 casos de não compatibilidade entre as duas instâncias gráficas construídas para comparação. Porém, somente em 42 casos esse diagnóstico é correto. Os falsos positivos de alterações dos objetos são associados a presença de obstrução por vegetação e prédios vizinhos e a falhas no processamento automático de segmentação e classificação. Na proposta de um sistema interativo, um operador poderia modificar sensivelmente os parâmetros associados às tolerâncias de decisão, com isso, reduzir tal superestimativa.

\section{REFERÊNCIAS BIBLIOGRÁFICAS}

Borgefors, G. Distance transformations in digital images. Computer Vision, Graphics and Image Processing, 34 (1986), pp. 344-371.

Chinpanthana, N. Semantic Similarity Measure with Conceptual Graph-Based Image Annotations. International Conference on Advanced Computer Science Applications and Technologies (ACSAT), IEEE Proceedings. 26-28 Nov. 2012, 203 - 208

Comaniciu, D., Meer, P. Robust Analysis of Feature Spaces: Color Image Segmentation, http://www.caip.rutgers.edu/ meer/RIUL/PAPERS/feature.ps.gz, in Proceedings of CVPR'97, San Juan, Puerto Rico.

Comaniciu, D., Meer, P. Mean Shift: A Robust Approach Toward Feature Space Analysis. IEEE Transactions on Pattern Analysis and Machine Intelligence, 2002. v. 24, n. 5.

Gonçalves, G. A. Detecção Automática De Alterações Na Cartografia Cadastral Com Base Em Imagens De Câmaras Digitais, Tese de Doutorado, Programa de Pós-Graduação em Ciências Geodésicas, Universidade Federal do Paraná, Curitiba, PR, 2006.

Gonzalez, R. C. e Woods, R. E. Digital Image Processing. Prentice Hall; 3th edition, 2007.

Gregoire, N. e Bouillot, M. Hausdorff Distance between convex polygons, Computational Geometry Web project, 1998, in http://www.cgrl.cs.mcgill.ca/ godfried/teaching/cgprojects/98/normand/ main.html

Gu, I.Y.H.; Gui, V. Color Image Segmentation using Adaptive Mean Shift Filters. Proceedings of IEEE International Conference Image Processing, ICIP 2001.

Huttenlocher, D. P.; Klanderman, G. A., Rucklidge, W. J. Comparing images using the Hausdorff distance, IEEE Trans. Pattern Anal. Machine Intell. 15 (9) 850-863. 1993.

Huttenlocher, D. P.; Kedem, K.; Kleinberg, J. M. On dynamic Voronoi diagrams and the minimum Hausdorff distance for point sets under Euclidean motion in the plane, to be published in Proc. Eighth ACM Symp. Computat. Geometry, 1992.

Huttenlocher, D. P.; Kedem, K. Efficiently computing the Hausdorff distance for point sets under translation, in Proc. Sixth ACM Symp. Computat. Geometry, 1990, pp. 340-349.

Jensen, J. R.; Introductory Digital Image Processing: A Remote Sensing Perspective. PrenticeHall, 1996. 
Kalinic, H.; Loncaric, S. ; Bijnens, B. A novel image similarity measure for image registration. International Symposium on Image and Signal Processing and Analysis (ISPA). IEEE Proceedings, 4-6 Sept. 2011. Pg. 195 - 199

Machado, A. M. L. Extração Automática De Contornos De Edificações Utilizando Imagem Gerada Por Câmara Digital De Pequeno Formato E Dados Lidar. Tese de Doutorado. Curso de Pós-Graduação em Ciências Geodésicas, Universidade Federal do Paraná, 2006.

Mayer, H. Automatic Object Extraction from Aerial Imagery-A Survey Focusing on Buildings. Computer Vision and Image Understanding, Computer Vision and Image Understanding, Volume 74, Issue 2, Pag. 138-149. Maio 1999.

McKeown Jr., D. M.; Harvey, W. A. e Wixson, L. E. Automating Knowledge Acquisition for Aerial Image Interpretation. Department of Computer Science, Carnegie-Mellon University. Computer Vision, Graphics, and Image Processing 46, 37-81, 1989.

Orrite, C.; Herrero, J. E. Shape matching of partially occluded curves invariant under projective transformation. Computer Vision and Image Understanding, 34-64. p. 603-619, 2004.

Paglieroni, D. W. Distance transforms: Properties and machine vision applications. Comput. Vision Graphics Image Processing: Graphical Models Image Processing, vol. 54, no. 1, pp. 5674, 1992.

Somboonkaew, A.; Chitwong, S.; Cheevasuvit, F.; Dejhan, K. E Mitatha S. Segmentation on the Edge Preserving Smoothing Image. Asian Conference on Remote Sensing, 1999. (versão digital disponível em http://www.gisdevelopment.net/aars/acrs/index.htm)

Xiaozhou, Yu. Hausdorff Distance for Shape Matching. Lecture Notes disponível em: http://www.cemnet.ntu.edu.sg/presentation/slides_yuxiaozhou.pdf. 2005;

Zhang-lin Guo; Jing Tian. International Conference on Computational Intelligence and Security Workshops, 2007. CISW 2007. IEEE Proceedings. 15-19 Dec. 2007. Pg. 299 - 303.

Recebido em Setembro de 2015.

Aceito em Maio de 2016 